RU9687

\title{
Studies in the statistical and thermal properties of hadronic matter under some extreme conditions
}

\author{
K. C. Chase, A. Z. Mekjian and P. Meenakshisundaram \\ Department of Physics, Rutgers University \\ Piscataway, New Jersey 08854
}

(March 27, 2017)

\begin{abstract}
The thermal and statistical properties of hadronic matter under some extreme conditions are investigated using an exactly solvable canonical ensemble model. A unified model describing both the fragmentation of nuclei and the thermal properties of hadronic matter is developed. Simple expressions are obtained for quantities such as the hadronic equation of state, specific heat, compressibility, entropy, and excitation energy as a function of temperature and density. These expressions encompass the fermionic aspect of nucleons, such as degeneracy pressure and Fermi energy at low temperatures and the ideal gas laws at high temperatures and low density. Expressions are developed which connect these two extremes with behavior that resembles an ideal Bose gas with its associated Bose condensation. In the thermodynamic limit, an infinite cluster exists below a certain critical condition in a manner similar to the sudden appearance of the infinite cluster in percolation theory. The importance of multiplicity fluctuations is discussed and some recent data from the EOS collaboration on critical point behavior of nuclei can be accounted
\end{abstract}


for using simple expressions obtained from the model.

25.70.Pq, 21.65.+f, 05.70.Ce, 05.70.Jk

Typeset using REVTEX 


\section{INTRODUCTION}

One of the goals of nuclear physics is to characterize the thermal properties of nuclear matter, described by its equation of state. Investigating heavy ion collisions offers one experimental method of probing nuclei away from the typical low temperature and density, and a number of experiments are underway studying the breakup of nuclei, contributing greatly to our understanding [1-6]. Several theoretical investigations of these properties have been instigated, thermal [7 10, statistical [11 15 transport [16 20] and percolative [21 26] models all offering insights into the properties of fragmenting nuclei.

In a previous set of papers [27 30] an exactly solvable canonical ensemble model was developed for studying the multifragmentation of nuclei induced by high energy collisions. Detailed properties of the cluster distributions such as inclusive yields and correlations were explored. In this paper, we study the statistical and thermal properties of nuclei using the same model, extending results presented in [31]. This model gives a unified description of multifragmentation phenomena and the thermodynamic properties of hadronic matter, such as its equation of state and the nuclear compressibility. Simple expressions for these quantities are obtained which extrapolate or connect between a low temperature nearly degenerate Fermi gas to a high temperature ideal Maxwell-Boltzmann gas of nucleons. Importantly, the effects of the fragmentation degrees of freedom on the thermodynamics is manifestly included. Questions related to very high temperatures and particle and anti-particle production will be developed in a subsequent paper.

\section{MODEL STUDIES}

Given the partition function $Z_{A}(V, T)$ for a system of $A$ nucleons at a given volume $V$ and temperature $T$ we can determine the thermodynamic functions by taking various partial derivatives, i.e. the internal energy and pressure are given by $U=k_{B} T^{2}\left(\frac{\partial}{\partial T} \ln Z_{A}\right)_{V}$,

$P=k_{B} T\left(\frac{\partial}{\partial V} \ln Z_{A}\right)_{T}$. Additional partial derivatives lead to such important quantities 
as the specific heat and isothermal compressibility, $C_{V}=\left(\frac{\partial U}{\partial T}\right)_{V}, \frac{1}{\kappa_{T}}=-\left(V \frac{\partial P}{\partial V}\right)_{T}$, and thermodynamic potentials such as the entropy and Gibbs free energy can be related to these quantities, $S=U / T+k_{B} \ln Z_{A}, G=-k_{B} T \ln Z_{A}+P V$. Our interest of course is not in the partition function itself, but rather in these derived functions, and as such the partition function is merely a means to that end.

This section constructs a partition function for fragmenting nuclear matter. It does so by investigating the thermodynamic functions at both low and high temperatures and densities. At low temperature/high density, the nucleons coalesce into a single nucleus which can be modeled as a degenerate Fermi gas with Skyrme interactions. At high temperature/low density, the nucleons evaporate into a Maxwell-Boltzmann gas of individual nucleons. With these results firmly in mind, a statistical fragmentation model is introduced whose parameters depend on the thermodynamics in a way consistent with the high and low temperature limits, yet allowing more complicated fragmentation patterns at intermediate temperatures. RHS always diverges in the infinite limit The remaining freedom in the model is eliminated by assuming that the cluster yields follow a power law. Interestingly these minimal assumptions lead to a model surprisingly rich in physics as we shall see in the next section.

\section{A. Low temperature behavior and ideal Fermi gas laws}

At zero temperature, the nucleus can be treated as a degenerate Fermi gas, with Fermi momentum $p_{F}$ given by

$$
g_{S, I} \frac{4}{3} \pi p_{F}^{3}=h^{3} \rho
$$

where $g_{S, I}=4$ is the spin-isospin degeneracy factor and $\rho=A / V$ is the density. At normal density $\rho_{0}=A / V_{0}=0.17$ nucleons $\mathrm{fm}^{-3}$, nucleons are non-relativistic and the Fermi energy

and momentum are related by $\varepsilon_{F}=p_{F}^{2} / 2 m$. From the above expression for the Fermi momentum, we see that $\varepsilon_{F} \propto \rho^{2 / 3}$. At low temperatures, the total kinetic energy for such a Fermi gas is given by 32 


$$
E_{K}=\frac{3}{5} A \varepsilon_{F}\left(1+\frac{5 \pi^{2}}{12}\left(\frac{k_{B} T}{\varepsilon_{F}}\right)^{2}\right) .
$$

However, the nucleons do interact. Interactions between the nucleons can be taken into account by using a density dependent Skyrme interaction [33,34. In this approach, the internal energy of a nucleus is given by

$$
U_{\mathrm{LT}}=E_{K}-A\left(a_{0} \frac{\rho}{\rho_{0}}-a_{3}\left(\frac{\rho}{\rho_{0}}\right)^{1+\sigma}\right),
$$

where $a_{0}$ and $a_{3}$ are Skyrme parameters (for simplicity, temperature independent) determined by fixing $a_{V}(\rho)=-U_{\mathrm{LT}}(T=0) / A$ the binding energy per particle at zero temperature

$$
a_{V}(\rho)=-\frac{3}{5} \epsilon_{F}(\rho)+a_{0}\left(\frac{\rho}{\rho_{0}}\right)-a_{3}\left(\frac{\rho}{\rho_{0}}\right)^{1+\sigma}
$$

to the empirical value for $a_{V}$ at $\rho=\rho_{0}$ (about $8.0 \mathrm{MeV} /$ nucleon at zero temperature) and having this as the maximum of $a_{V}$. The density dependent repulsive term should appear with a higher power of $\rho$ than the attractive part, so that the nucleus does not collapse, and thus requires $\sigma>0$. Commonly used values for $\sigma$ are $\sigma=1$ (three body interaction) and $\sigma=2 / 3$ (finite range term) 35.

Having specified the internal energy, we can now determine any other thermodynamic function. The specific heat $C_{V}=\left(\frac{\partial U}{\partial T}\right)_{V}$, and entropy $S=\int{ }^{T} C_{V} / T^{\prime} d T^{\prime}$ are therefore

$$
\left(\frac{C_{V}}{k_{B}}\right)_{\mathrm{LT}}=\left(\frac{S}{k_{B}}\right)_{\mathrm{LT}}=A \frac{\pi^{2}}{2}\left(\frac{k_{B} T}{\varepsilon_{F}}\right) .
$$

The partition function can now be determined from $Z_{A}=\exp \left\{(S T-U) / k_{B} T\right\}$, which gives

$$
Z_{A}=y^{A}
$$

where

$$
y=\exp \left\{\frac{a_{V}}{k_{B} T}+\frac{k_{B} T}{\varepsilon_{0}}\right\},
$$

and $\varepsilon_{0}=\frac{4}{\pi^{2}} \varepsilon_{F}$. ¿From this partition function it is easy to determine the pressure and compressibility. 


$$
\begin{aligned}
P_{\mathrm{LT}}= & \frac{2}{5} \rho \varepsilon_{F}(\rho)\left(1+\frac{5 \pi^{2}}{12}\left(\frac{k_{B} T}{\varepsilon_{F}}\right)^{2}\right) \\
& -a_{0} \rho \frac{\rho}{\rho_{0}}+(1+\sigma) a_{3} \rho\left(\frac{\rho}{\rho_{0}}\right)^{1+\sigma}, \\
\left(\frac{1}{\kappa_{T}}\right)_{\mathrm{LT}}= & \frac{2}{3} \rho \varepsilon_{F}\left(1+\frac{\pi^{2}}{12}\left(\frac{k_{B} T}{\varepsilon_{F}}\right)^{2}\right) \\
& -2 a_{0} \frac{\rho^{2}}{\rho_{0}}+(1+\sigma)(2+\sigma) a_{3} \rho\left(\frac{\rho}{\rho_{0}}\right)^{1+\sigma} .
\end{aligned}
$$

\section{B. High temperature, low density ideal Maxwell-Boltzmann gas laws}

At high temperature and/or low density, but below meson and particle-antiparticle production thresholds, the hadronic properties are that of an ideal Maxwell-Boltzmann gas, with

$$
\begin{aligned}
U_{\mathrm{HT}} & =\frac{3}{2} A k_{B} T, \\
\left(\frac{C_{V}}{k_{B}}\right)_{\mathrm{HT}} & =\frac{3}{2} A, \\
P_{\mathrm{HT}} & =\rho k_{B} T, \\
\left(\frac{1}{\kappa_{T}}\right)_{\mathrm{HT}} & =\rho k_{B} T, \\
\left(\frac{S}{k_{B}}\right)_{\mathrm{HT}} & =A \ln \left\{e^{5 / 2} \frac{V}{A \lambda_{T}^{3}} g_{S, I}\right\},
\end{aligned}
$$

where the entropy $S$ is given by the Sackur-Tetrode law and $\lambda_{T}=h / \sqrt{2 \pi m k_{B} T}$. We expect that nuclei vaporize into individual nucleons at $k_{B} T \gg a_{V}$, the binding energy per particle. The partition function consistent with these thermodynamic functions is $Z_{A}=x^{A} / A$ !, where $x=V / \lambda_{T}^{3}$.

\section{Hadronic matter at moderate temperatures and densities and ideal Bose gas-like structure}

In the region between the ideal Fermi gas limit and the ideal Maxwell-Boltzmann gas limit, the effects of the fragmentation of the initial nucleus must be taken into account 
when considering thermodynamic issues. To account for these effects, we employ a model developed initially to study multifragmentation phenomena [36]. This model has the correct high and low temperature limits, as determined in the previous sections, but has features similar to that of a Bose gas in the intermediate range.

To describe the fragmentation of a nucleus into all possible modes of breakup, a weight is given to each possibility. The weight chosen is

$$
W(\mathbf{n})=\prod_{k \geq 1} \frac{x_{k}^{n_{k}}}{n_{k} !}=\prod_{k \geq 1} \frac{1}{n_{k} !}\left(\frac{x y^{k-1}}{\beta_{k}}\right)^{n_{k}},
$$

where $x$ and $y$ are functions of the thermodynamic variables $(V, T), \beta_{k}$ is the cluster size dependence of the weight and $\mathbf{n}=\left(n_{1}, n_{2}, \ldots\right)$ is the fragmentation vector, with $n_{k}$ the number of fragments with $k$ nucleons such that $\sum_{k} k n_{k}=A$. With this choice of weight the canonical partition function $Z_{A}=\sum_{\mathbf{n}} W(\mathbf{n})$ is a polynomial in $x, y$, given by

$$
Z_{A}(x, y)=\sum_{m=1}^{A} Z_{A}^{(m)}(\vec{\beta}) x^{m} y^{A-m},
$$

with $m=\sum_{j} n_{j}$ the multiplicity. Given the partition function, all thermodynamic properties of the model can be obtained, as well as ensemble averages. For example, the mean number of clusters of size $k$ is

$$
\left\langle n_{k}\right\rangle=\frac{x y^{k-1}}{\beta_{k}} \frac{Z_{A-k}(x, y)}{Z_{A}(x, y)} .
$$

The partition functions themselves can be obtained using a recursive procedure defined by the constraint $\sum_{k} k\left\langle n_{k}\right\rangle=A$, which gives

$$
Z_{A}(x, y)=\frac{1}{A} \sum_{k=1}^{A} k \frac{x y^{k-1}}{\beta_{k}} Z_{A-k}(x, y),
$$

where $Z_{0}(x, y)=1$. The whole procedure is easily implemented by computer.

The parameters $x, y$ determine the thermodynamic aspects of the models and as such need to be determined. They are most easily determined by considering the partition function at high and low multiplicity. When the multiplicity is large $\langle m\rangle \approx A$, and $Z_{A} \approx Z_{A}^{(A)} x^{A}$. Clearly this is the high temperature limit, and $x$ is simply the ideal gas $x$ introduced in section IIB, given by 


$$
x=\frac{V}{\lambda_{T}^{d}}
$$

and involves the volume of the system $V$, the thermal wavelength $\lambda_{T}$, and the dimensionality of the system $d$. The term $x^{m}$ in the weight arises from the thermal motion of each fragment. Since the overall motion is zero, this should be replaced by $x^{m-1}$ to reflect the center of momentum constraint. When the multiplicity is small $\langle m\rangle \approx 1$, and $Z_{A} \approx Z_{A}^{(1)} x y^{A-1}$. Assuming that the $x$ parameter is removed by the conservation of momentum constraint describe above, this shows $y$ to be given by Eq. (7). A correction to the internal excitations however needs to be made at high temperatures to reflect their finite lifetimes. Koonin and Randrup [37] argue that a simple cutoff is effective, which results in

$$
y=\exp \left\{\frac{a_{V}}{k_{B} T}+\frac{k_{B} T}{\varepsilon_{0}} \frac{T_{0}}{T+T_{0}}\right\} .
$$

Here $a_{V}$ is the binding energy per particle, $\varepsilon_{0}$ is the level spacing parameter (related to the

Fermi energy) and $T_{0}$ is the cutoff temperature. So the term $y^{A-m}$ in the weight arises from the binding and internal excitations of each fragment.

The only parameter now to determine is $\beta_{k}$. Since the mass yield distributions are often well represented by a power law [38, we choose $\beta_{k}$ simply to reproduce this important experimental fact. If $\beta_{k}=k^{\tau}$, then in the grand canonical limit $\left\langle n_{k}\right\rangle \sim k^{-\tau}$. Typically $\tau$ is found between two and three, and we have chosen $\tau=2.5$ as representative for calculations made in this paper.

\section{THERMODYNAMIC PROPERTIES OF HADRONIC MATTER}

In this section we consider the various fundamental thermodynamic functions of fragmenting nuclei, namely the pressure, compressibility and Gibbs free energy (section ПIA), the internal energy and specific heat (section [IIB), and the entropy (section IIIQ). Interesting features of the functions are explored, although a detailed examination of their origin is postponed till the next section. A brief accounting of such properties has already been given 31. 
Since the partition function can be expressed succinctly as a function of $x, y$, derivatives of $\ln Z_{A}$ with respect to these variables will appear and reappear in computations of thermodynamic functions. Fortunately for this model, derivatives with respect to $x, y$ yield expectation values of the multiplicity, i.e. $x \frac{\partial}{\partial x} \ln Z_{A}=\langle m\rangle, y \frac{\partial}{\partial y} \ln Z_{A}=A-\langle m\rangle$, $\left(x \frac{\partial}{\partial x}\right)^{2} \ln Z_{A}=\left(y \frac{\partial}{\partial y}\right)^{2} \ln Z_{A}=\operatorname{Var}(m)=\left\langle m^{2}\right\rangle-\langle m\rangle^{2}$. A general derivation of these identities is included in appendix A.

\section{A. The hadronic equation of state and a van der Waals like structure}

The hadronic equation of state is of much interest since the pressure and incompressibility reflect the behavior of matter at fixed temperatures and varying volumes. A simple expression can be obtained for this equation of state by using the $P=k_{B} T \frac{\partial}{\partial V} \ln Z_{A}$ and the partition function of Eq. (16) as determined from the weight in Eq. (15), giving

$$
P=\frac{\langle m\rangle}{A} P_{\mathrm{HT}}+\left(1-\frac{\langle m\rangle}{A}\right) P_{\mathrm{LT}}^{\prime}
$$

where $\langle m\rangle=\sum_{k}\left\langle n_{k}\right\rangle$ is the mean multiplicity, $P_{\mathrm{LT}}, P_{\mathrm{HT}}$ are the low and high temperature limits defined in sections $\Pi \mathrm{A}$, ПB and $P_{\mathrm{LT}}^{\prime}=P_{\mathrm{LT}}-\frac{2}{3} \rho \varepsilon_{0}\left(\frac{k_{B} T}{\varepsilon_{0}}\right)^{2} \frac{T}{T+T_{0}}$ is simply the cutoff corrected low temperature pressure. The above equation shows the importance of the mean multiplicity in the behavior of $P$. For low $x / y,\langle m\rangle \approx 1$, and the pressure reduces to the Fermi gas and Skyrme pressure $P_{\mathrm{LT}}$. At high $x / y,\langle m\rangle \approx A$ and the ideal MaxwellBoltzmann gas pressure $P_{\mathrm{HT}}$ in $P$ dominates. Thus the result of Eq. (21) connects the two extremes in a simple analytic way. Figure [1(a) plots the pressure for several temperatures for $\beta_{k}=k^{5 / 2}$.

The low temperature component of the pressure is quite interesting. It is responsible for the pressure rising as the volume increases over a range of volumes at low enough temperatures, a feature characteristic of a van der Waals gas or a liquid-gas phase instability [8]. It arises due to the nuclear interactions. To see this phase instability more clearly, we can calculate the isothermal compressibility from the equation of state, since $\frac{1}{\kappa_{T}}=\left(\rho \frac{\partial P}{\partial \rho}\right)_{T}$, a little effort gives us 


$$
\begin{aligned}
\frac{1}{\kappa_{T}}= & \frac{\langle m\rangle}{A} P_{\mathrm{HT}}+\left(1-\frac{\langle m\rangle}{A}\right) \rho \frac{\partial P_{\mathrm{LT}}^{\prime}}{\partial \rho} \\
& -\frac{\operatorname{Var}(m)}{A} \frac{\left(P_{\mathrm{HT}}-P_{\mathrm{LT}}^{\prime}\right)^{2}}{P_{\mathrm{HT}}} .
\end{aligned}
$$

At large values of $x / y,\langle m\rangle \approx A, \operatorname{Var}(m) \approx 0$ and $\frac{1}{\kappa_{T}} \rightarrow P_{\mathrm{HT}}$, the ideal gas limit. At low $x / y,\langle m\rangle \approx 1, \operatorname{Var}(m) \approx 0$ and $\frac{1}{\kappa_{T}} \rightarrow P_{\mathrm{LT}}^{\prime}$, an ideal Fermi gas with Skyrme interaction limit. In between, there is an effect due to the fluctuation in the mean number of clusters. The behavior of the compressibility is shown in Fig. 1(b). From the figure, the phase instability appears first at $\rho=0.5 \rho_{0}, T=24 \mathrm{MeV}$, the point where $\frac{1}{\kappa_{T}}$ and its derivative are both zero.

Another method of seeing this instability is to consider the Gibbs free energy $G=$ $-k_{B} T \ln Z_{A}+P V$. Figure 2 shows that $G$ vs. $P$ is multivalued below a critical temperature, a clear indication of a phase instability.

In nuclear physics, one usually refers to the incompressibility $\kappa=\frac{9}{\rho \kappa_{T}}$, which if $\varepsilon_{F} \approx 37$ $\mathrm{MeV}$ gives at $T=0$

$$
\kappa=96+144 \sigma \mathrm{MeV}
$$

For $\sigma=1$, this model $\kappa=240 \mathrm{MeV}$, consistent with the usually quoted value for the incompressibility.

\section{B. Internal energy, specific heat and the ideal Bose gas}

The energy and specific heat are important quantities also, especially when the volume is fixed and the temperature is changing. The internal energy decomposes into low and high temperature components just as the pressure does, namely

$$
U=\frac{\langle m\rangle}{A} U_{\mathrm{HT}}+\left(1-\frac{\langle m\rangle}{A}\right) U_{\mathrm{LT}}^{\prime},
$$

where $U_{\mathrm{LT}}^{\prime}=U_{\mathrm{LT}}-A \varepsilon_{0}\left(\frac{k_{B} T}{\varepsilon_{0}}\right)^{2} \frac{T\left(T+2 T_{0}\right)}{\left(T+T_{0}\right)^{2}}$ is simply the cutoff corrected low temperature energy. Figure 3(a) plots this behavior for a number of densities, which reveals little except for the monotonic increasing nature of the energy. 
The specific heat on the other hand is more revealing. Using $C_{V}=\left(\frac{\partial U}{\partial T}\right)_{V}$, one arrives at

$$
\begin{aligned}
\frac{C_{V}}{k_{B}}= & \langle m\rangle \frac{d}{2}+(A-\langle m\rangle) \frac{2 k_{B} T}{\varepsilon_{0}}\left(\frac{T_{0}}{T+T_{0}}\right)^{3} \\
& +\operatorname{Var}(m)\left(\frac{d}{2}+\frac{a_{V}}{k_{B} T}-\frac{k_{B} T}{\varepsilon_{0}}\left(\frac{T_{0}}{T+T_{0}}\right)^{2}\right)^{2}
\end{aligned}
$$

and Fig. 3(b) illustrates the behavior of $C_{V}$ for $d=3, \beta_{k}=k^{5 / 2}$. The ideal gas limit is seen in the first term $\langle m\rangle \frac{d}{2}$. The low temperature Fermi gas result is contained in the second term $(A-\langle m\rangle)\left(2 k_{B} T / \varepsilon_{0}\right)$. The last term involving the multiplicity fluctuations gives rise to a peak in $C_{V} / k_{B}$ when $d>2$.

The variance induced peak is quite interesting. As we shall see in section IVA, it is analogous to condensation in an ideal Bose-Einstein gas, a critical transition which has been extensively studied.

\section{The entropy of nuclei}

The entropy of fragmenting nuclei can be obtained from the relation $S=\frac{\partial}{\partial T} k_{B} T \ln Z_{A}$ which gives

$$
\begin{aligned}
\frac{S}{k_{B}}= & \ln Z_{A}+\langle m\rangle \frac{d}{2} \\
& +(A-\langle m\rangle)\left(\frac{-a_{V}}{k_{B} T}+\frac{k_{B} T}{\varepsilon_{0}}\left(\frac{T_{0}}{T+T_{0}}\right)^{2}\right) .
\end{aligned}
$$

When $\langle m\rangle \approx 1$, the entropy is that of a nearly degenerate Fermi gas of $A$ nucleons confined to a cluster of size $A$, plus a contribution from the motion of this cluster which is coupled to a heat bath in the canonical ensemble. The nearly degenerate Fermi gas entropy is given by Eq. (5) where cutoff effects are excluded. Including it gets

$$
\frac{S}{k_{B}}=A \frac{\pi^{2}}{2}\left(\frac{k_{B} T}{\varepsilon_{0}}\right)\left(1-\frac{T}{2\left(T+T_{0}\right)}\right) .
$$

The cutoff correction is of the same order as the next higher order correction. When $\langle m\rangle \approx A$, $S$ reduces to the Sackur-Tetrode law of Eq. (14). In the intermediate regime, their is a critical point where a latent heat must be overcome, causing a change in volume. 
Figure 1 is a plot of $S / k_{B} A$ vs. $k_{B} T$. In the plot, the contribution from the thermal motion of the largest cluster has been subtracted out so that $S \rightarrow 0$ as $\langle m\rangle \rightarrow 1$. This is equivalent to requiring the total momentum of the system to be zero, a correction consistently made throughout these calculations.

\section{CRITICAL POINT BEHAVIOR AND PARALLELS WITH OTHER MODELS}

The thermodynamic functions strongly suggest a critical point, which we determine explicitly in this section. To start, let us work out some properties of the cluster distributions in the grand canonical ensemble. Here the partition function is given by $\mathcal{Z}=\sum_{A} z^{A} Z_{A}(x, y)=\exp \sum_{k}(x / y) z^{k} / k^{\tau}$ where $\beta_{k}=k^{\tau}$ and $z=z^{\prime} y=e^{\mu / k_{B} T}$ is the chemical potential. In this limit $\left\langle n_{k}\right\rangle$ is simply

$$
\left\langle n_{k}\right\rangle=\frac{x}{y} \frac{z^{k}}{k^{\tau}}
$$

Since $A=\sum_{k} k\left\langle n_{k}\right\rangle$, we have

$$
\frac{A}{x^{\prime}}=\sum_{k=1}^{A} z^{k} k^{1-\tau}=g_{\tau-1}(z)
$$

with $x^{\prime}=x / y, x, y$ as defined earlier and $g_{n}(z)=\sum_{k>0} k^{-n} z^{k}$. Notice that $g_{n-1}(z)=$ $z \partial g_{n} / \partial z$ and that for $z \approx 1$

$$
g_{n}(z)=\nu^{n-1} \Gamma(1-n)+\sum_{k \geq 0} \zeta(n-k)(-1)^{k} \frac{\nu^{k}}{k !},
$$

where $\nu=-\ln z$, a result due to London [39].

The LHS of Eq. (28) is always finite in the thermodynamic limit. If $z>1$, the RHS always diverges in the infinite limit $A \rightarrow \infty$, and the equation cannot hold. At $z=1$, $\sum_{k} k^{1-\tau}=\zeta(\tau-1)$, the Riemann zeta function, which is finite only if $\tau>2$. If $A / x^{\prime} \leq \zeta(\tau-1)$ then a $z \leq 1$ can be found that satisfies the last constraint equation including all terms in the sum. Otherwise, the grand canonical expression breaks down, which defines a critical point 


$$
\frac{A}{x_{c}^{\prime}}=\zeta(\tau-1)
$$

The failure of the grand canonical ensemble is due to the appearance of an infinite cluster, i.e. of size $\alpha A$ where $\alpha$ is nonzero even as $A$ tends to infinity. More specifically, when $A / x^{\prime}$ exceeds $\zeta(\tau-1)$, an infinite cluster exists, absorbing enough mass so that the remaining mass is distributed grand canonically with $z=1$. If $A / x^{\prime}$ is less that $\zeta(\tau-1)$, an infinite cluster does not exist.

As we have already seen, the multiplicity and its fluctuations play an important role in the thermodynamic functions. So how does the critical point affect the multiplicity and its fluctuations? The multiplicity $\langle m\rangle=\sum_{k}\left\langle n_{k}\right\rangle$ is given simply by

$$
\frac{\langle m\rangle}{A}=\left\{\begin{array}{ll}
\frac{x^{\prime} g_{\tau}(1)}{A} & x^{\prime}<x_{c}^{\prime} \\
\frac{x^{\prime} g_{\tau}(z)}{A} & x^{\prime}>x_{c}^{\prime}
\end{array} .\right.
$$

The variance of the multiplicity $\operatorname{Var}(m)=x \frac{\partial}{\partial x}\langle m\rangle$ is given by

$$
\frac{\operatorname{Var}(m)}{A}=\left\{\begin{array}{ll}
\frac{x^{\prime} g_{\tau}(1)}{A} & x^{\prime}<x_{c}^{\prime} \\
\frac{x^{\prime} g_{\tau}(z)}{A}-\frac{g_{\tau-1}(z)}{g_{\tau-2}(z)} & x^{\prime}>x_{c}^{\prime}
\end{array} .\right.
$$

Figure 5(a),(b) plots $\operatorname{Var}(m)=\left\langle m^{2}\right\rangle-\langle m\rangle^{2}$. vs. $x^{\prime}$ and $\langle m\rangle$ respectively for both the canonical and grand canonical solutions. The discontinuity in the slope of $\operatorname{Var}(m)$ at the critical point is apparent from the figure. The behavior of $\operatorname{Var}(m)$ vs. $x$ shows a cusp-like behavior. For finite systems, $\operatorname{Var}(m)$ has a rounded peak. As we shall see in the next section, this behavior is related to the ideal Bose-Einstein gas condensation.

Is this critical behavior consistent with the experimental situation in nuclear collisions? At the critical point $x=x_{c}$, where $\operatorname{Var}(m)$ has a cusp, $\langle m\rangle=\langle m\rangle_{c}$ with

$$
\frac{\langle m\rangle_{c}}{A}=\frac{\zeta(\tau)}{\zeta(\tau-1)}
$$

The EOS collaboration experiment determined that for gold multifragmentation, the charge multiplicity $\langle m\rangle_{c}=26 \pm 1$. Using the above expression and $Z=79$ for $A$, results in a critical exponent $\tau=2.262 \pm 0.013$. Using a percolation theoretic analysis, they arrived at 
a somewhat different $\tau=2.14 \pm 0.06$. The two results are sufficiently close to suggest that the connection between the critical multiplicity and critical exponent $\tau$ 40 may not differ greatly from this simple model.

\section{A. Ideal Bose-Einstein gas laws}

Let us now compare these results with the ideal Bose gas of $A$ particles. The results can be found in Huang [32] for the case $d=3$ dimensions, and generalizing to arbitrary dimensions is fairly straightforward.

In the grand canonical ensemble, the ideal Bose gas of $A$ particles moving in a volume $V$ at a temperature $T$ has a fugacity $z=\exp \left\{\mu / k_{B} T\right\}$ determined by

$$
\frac{A}{V}=\frac{1}{\lambda_{T}^{d}} g_{d / 2}(z)+\frac{1}{V} \frac{z}{1-z}
$$

The critical temperature for a particular density and temperature occurs when $z \rightarrow 1$, $V \rightarrow \infty$, which implies

$$
\rho_{c}=\frac{A}{V}=\frac{1}{\lambda_{c}^{d}} g_{d / 2}(1)
$$

since above the critical point, the second term can be neglected in the infinite volume limit. Recalling that $x=V / \lambda_{T}^{d}$, this condition is identical to the fragmentation case if we replace $x$ by $x^{\prime}$ and $\tau$ by $1+d / 2$.

The energy, specific heat, pressure and incompressibility above and below the critical point are given by

$$
\begin{aligned}
\frac{U}{k_{B} T} & =\left\{\begin{array}{l}
\frac{d}{2} \frac{V}{\lambda^{d}} g_{1+d / 2}(z) T>T_{c} \\
\frac{d}{2} \frac{V}{\lambda^{d}} g_{1+d / 2}(1) T<T_{c}
\end{array}\right. \\
\frac{C_{V}}{k_{B}} & =\left\{\begin{array}{ll}
\frac{d(d+2)}{4} \frac{V}{\lambda^{d}} g_{1+d / 2}(z)-\frac{d^{2}}{4} \frac{g_{d / 2}(z)}{g_{d / 2-1}(z)} & T>T_{c} \\
\frac{d(d+2)}{4} \frac{V}{\lambda^{d}} g_{1+d / 2}(1) & T<T_{c}
\end{array},\right. \\
P & = \begin{cases}\frac{k_{B} T}{\lambda^{d}} g_{1+d / 2}(z) & T>T_{c} \\
\frac{k_{B} T}{\lambda^{d}} g_{1+d / 2}(1) & T<T_{c}\end{cases}
\end{aligned}
$$




$$
\frac{1}{\kappa_{T}}=\left\{\begin{array}{ll}
\frac{k_{B} T}{\lambda^{d}} \frac{g_{d / 2}^{2}(z)}{g_{d / 2-1}(z)} & T>T_{c} \\
0 & T<T_{c}
\end{array} .\right.
$$

Both above and below the critical point these results agree with the fragmentation case $y=1, \tau=1+d / 2$ if we apply Eqs. (31) and (32).

This agreement can be understood by noting that the weight given to the ideal Bose gas in $d$ dimensions in a Mayer cluster expansion [32] is given by Eq. (15) with parameter vector

$$
x_{k}=\frac{x}{k^{1+d / 2}}+\frac{1}{k}
$$

At large $x$ (i.e above the critical point) the second term is negligible and the model reverts to the one considered here. Below the critical point, the large cluster formed contributes little to the thermodynamic functions, allowing agreement to continue.

The nature of the transition is first order as expected for a Bose-Einstein like condensation. The particles which have accumulated into the zero momentum mode contribute no pressure and have no volume or entropy in the thermodynamic limit. The pressure due to the balance of particles is constant for a given temperature (i.e. independent of volume). A latent heat per particle of

$$
L=\left(\frac{g_{1+d / 2}(1)}{g_{d / 2}(1)}\right) \frac{d+2}{2} k_{B} T,
$$

accompanies the transition and of course is the heat $T \Delta S$ which must be spent in moving a particle from the vapor phase which has entropy to the condensed phase which has no entropy. The latent heat of Eq. (41) is the result of the change in volume along the constant pressure Maxwell line at a fixed $T$. The volume change is

$$
V_{c}=\frac{A}{\lambda_{T}^{d}} \frac{1}{g_{d / 2}(1)} .
$$

The entropy change can be obtained from the Clausius-Clapeyron equation $d P_{c} / d T=S_{c} / V_{c}$ to give

$$
\frac{S_{c}}{k_{B}}=\frac{d+2}{2} A \frac{g_{1+d / 2}(1)}{g_{d / 2}(1)},
$$


which is consistent with $L=k_{B} T S_{c}$ as expected. The fraction of the number of particles in the Bose condensate (zero momentum mode) is

$$
\frac{N_{c}}{N}=1-\left(\frac{T}{T_{C}}\right)^{d / 2},
$$

for $T \leq T_{c}$.

In nuclear fragmentation, this condensation is also present, but the clusterization occurs in real space instead of momentum space. Rather than the number of particles in the zero momentum mode signaling a phase transition, the number of particles in the largest cluster plays the same role.

\section{B. Critical point behavior and percolation theory}

Percolation models 41] have been used to describe nuclear fragmentation with percolation clusters corresponding to nuclear clusters arising from the collision process. The percolation cluster distribution is given in terms of the percolation probability $p$ and percolation threshold probability $p_{c}$ above which an infinite cluster exists. Specifically,

$$
\left\langle n_{k}\right\rangle=\frac{1}{k^{\tau}} f\left(\left(p-p_{c}\right) / p_{c}\right)
$$

where $f(x)$ is a scaling function. Percolation models have also suggested useful methods of analyzing nuclear fragmentation data such as Campi plots [42].

The model in section \1 has some features that are similar with the percolation model. First, only one parameter $x^{\prime}=x / y$ describes the distribution, the analog of $p$ in percolation theory. Second, below a critical point $x_{c}^{\prime}$, the fraction of particles found in the largest cluster is finite, even if the number of particles tends to infinity, a situation familiar from percolation theory where for $p>p_{c}$ an infinite cluster exists, while for $p<p_{c}$ it can not exist. For the model of section $\mathbb{I}$ this can be seen by analogy with the Bose condensate, where a finite fraction of particles end up in the zero momentum state. In this nuclear fragmentation

model, a finite fraction of the mass ends up in the largest cluster. Specifically if $k_{\max }$ is the size of the largest cluster in each event, then in the infinite $A$ limit 


$$
\frac{\left\langle k_{\max }\right\rangle}{A}=\left\{\begin{array}{ll}
1-\frac{x}{x_{c}}=1-\frac{\langle m\rangle}{\langle m\rangle_{c}} & x<x_{c} \\
0 & x>x_{c}
\end{array} .\right.
$$

This threshold behavior of $\left\langle k_{\max }\right\rangle / A$ as expected exactly parallels Bose-Einstein condensation. Figure 6 shows the behavior of $\left\langle k_{\max }\right\rangle$ and its variance for some a range of $x^{\prime}$ for $\beta_{k}=k^{5 / 2}$. Due to scaling behavior $\left\langle k_{\max }\right\rangle / A$ for various $A$ 's approach the single curve shown above.

At the critical point, $\left\langle n_{k}\right\rangle \sim k^{-\tau}$ where $\tau>2$ can be chosen when specifying the original weight. The critical exponent $\tau$ for percolation is 2.21 .

\section{CONCLUSION AND SUMMARY}

In this paper, hadronic matter under some extreme conditions was investigated using an exactly solvable model which can describe both the fragmentation of nuclei and the thermal properties in a unified way. Three distinct types of behavior characterize hadronic matter. At low temperatures, the fermionic aspects of the system are apparent through such quantities as Fermi degeneracy pressure and Fermi energy. At high temperature and low density, ideal gas laws are shown to characterize the system. Between these two limits, a regime exists in which the behavior of the fragmenting matter resembles an ideal Bose gas with its associated Bose-Einstein condensation. The consequences of Bose-Einstein condensation on the properties of hadronic matter were studied. Simple expressions were obtained for the nuclear equation of state, heat capacity, compressibility, entropy, energy and fragmentation yields which encompass these three regimes. The importance of multiplicity fluctuations in statistical models of nuclear fragmentation and its effect on various thermodynamic properties is stressed. Some recent data from the EOS collaboration on critical point behavior of hadronic matter is analyzed and simple expressions from the exactly solvable model are obtained which can account for some features of the data. 


\section{ACKNOWLEDGMENTS}

This work supported in part by the National Science Foundation Grant No. NSFPHY 92-12016 and by the Department of Energy Grant No. DE-FG02-96ER40987.

\section{APPENDIX A: MULTIPLICITY EXPECTATION VALUES}

Given a function $f(m)$ where $m$ is the multiplicity, it is often useful to compute $x \frac{\partial}{\partial x}\langle f(m(x, y))\rangle$. If $f(m)$ is a polynomial, the following identities can be used to compute $x \frac{\partial f}{\partial x}$

$$
\begin{aligned}
x \frac{\partial}{\partial x}\left\langle[m]_{k}\right\rangle & =\left\langle m[m]_{k}\right\rangle-\langle m\rangle\left\langle[m]_{k}\right\rangle, \\
x \frac{\partial}{\partial x}\left\langle m^{k}\right\rangle & =\left\langle m^{k+1}\right\rangle-\langle m\rangle\left\langle m^{k}\right\rangle, \\
x \frac{\partial}{\partial x}\langle m\rangle_{k} & =\langle m\rangle_{k+1}-k\langle m\rangle_{2}\langle m\rangle_{k-1} .
\end{aligned}
$$

where $[x]_{n}=\mathrm{x}(\mathrm{x}-1) \ldots(\mathrm{x}-\mathrm{n}+1)$ and $\langle m\rangle_{n}=$ nth cumulant moment of $\mathrm{m}$. The first is easily derived from the fact that $\left\langle[m]_{k}\right\rangle=\frac{x^{k}}{Z_{A}(x)} \frac{\partial^{k} Z_{A}}{\partial x^{k}}$. The second identity can be derived from the first by expanding $\left\langle m^{k}\right\rangle=\sum_{j=0}^{k}\left\{\begin{array}{l}k \\ j\end{array}\right\}\left\langle[m]_{k}\right\rangle$, where $\left\{\begin{array}{l}k \\ j\end{array}\right\}$ is the Stirling number of the second kind, combined with the additional identity $\left\langle[m]_{k}\right\rangle=\sum_{j=0}^{k}(-1)^{k-j}\left[\begin{array}{l}k \\ j\end{array}\right]\left\langle m^{k}\right\rangle$, where $\left[\begin{array}{l}k \\ j\end{array}\right]$ is the unsigned Stirling number of the first kind, and the orthogonality relations between the two Stirling numbers. The third can be derived from the second using the binomial expansion of $(m-\langle m\rangle)^{k}$.

These three identities are supplemented by three additional identities with $x \frac{\partial}{\partial x} \rightarrow-y \frac{\partial}{\partial y}$. 


\section{REFERENCES}

[1] J. Pochodzalla et al., Phys. Rev. Lett. 75, 1040 (1995), aLADIN Collaboration.

[2] P. Kreutz et al., Nucl. Phys. A 556, 672 (1993).

[3] S. Wang et al., Phys. Rev. Lett. 74, 2646 (1995), eOS Collaboration.

[4] K. Kwiatkowski et al., Phys. Rev. Lett. 74, 3756 (1995).

[5] D. Handzy et al., Phys. Rev. Lett. 75, 2916 (1995).

[6] M. L. Gilkes et al., Phys. Rev. Lett. 72, 1590 (1994), eOS Collaboration.

[7] L. Csernai and J. Kapusta, Phys. Rep. 131, 223 (1986).

[8] A. L. Goodman, J. I. Kapusta, and A. Z. Mekjian, Phys. Rev. C 30, 851 (1984).

[9] M. W. Curtin, H. Toki, and D. K. Scott, Phys. Lett. B 123, 289 (1983).

[10] G. F. Bertsch and P. J. Siemens, Phys. Lett. B 126, 9 (1983).

[11] G. F. Bertsch and S. Das Gupta, Phys. Rep. 160, 189 (1988).

[12] L. Moretto and G. Wozniak, in Prog. Part. Nucl. Phys., edited by A. Faessler (Pergamon Press, Oxford, 1988), Vol. 21, pp. 401-457.

[13] J. P. Bondorf et al., Phys. Rep. 257, 133 (1995).

[14] D. H. E. Gross, Rep. Prog. Phys. 53, 605 (1990).

[15] J. Pan and S. Das Gupta, Phys. Rev. C 51, 1384 (1995).

[16] G. F. Bertsch, S. Das Gupta, and H. Kruse, Phys. Rev. C 29, 673 (1984).

[17] H. H. Gan, S. J. Lee, and S. Das Gupta, Phys. Rev. C 36, 2365 (1987).

[18] J. Aichelin, Phys. Rep. 202, 233 (1991).

[19] E. Suraud, S. Ayik, M. Belkacem, and J. Stryjewski, Nucl. Phys. A 542, 141 (1992). 
[20] A. Bonasera and F. Gulminelli, Phys. Lett. B 275, 24 (1992).

[21] X. Campi and H. Krivine, Nucl. Phys. A 545, 161c (1992).

[22] J. Desbois, Nucl. Phys. A 466, 724 (1987).

[23] W. Bauer, U. Post, D. Dean, and U. Mosel, Nucl. Phys. A 452, 699 (1986).

[24] T. S. Biro, J. Knoll, and J. Richert, Nucl. Phys. A 459, 692 (1986).

[25] S. Das Gupta, C. Gale, and K. Haglin, Phys. Lett. B 302, 372 (1993).

[26] J. B. Elliott et al., Phys. Rev. C 49, 3185 (1994), eOS Collaboration.

[27] A. Z. Mekjian, Phys. Rev. Lett. 64, 2125 (1990).

[28] A. Z. Mekjian, Phys. Rev. C 41, 2103 (1990).

[29] S. J. Lee and A. Z. Mekjian, Phys. Lett. A 149, 7 (1990).

[30] K. C. Chase and A. Z. Mekjian, Phys. Rev. C 49, 2164 (1994).

[31] K. C. Chase and A. Z. Mekjian, Phys. Rev. Lett. 75, 4732 (1995).

[32] K. Huang, Statistical Mechanics, 2nd ed. (John Wiley \& Sons, New York, 1987).

[33] D. Vautherin and D. M. Brink, Phys. Rev. C 5, 626 (1972).

[34] T. H. R. Skyrme, Nucl. Phys. 9, 615 (1959).

[35] H. Jaqaman, A. Z. Mekjian, and L. Zamick, Phys. Rev. C 27, 2782 (1983).

[36] S. J. Lee and A. Z. Mekjian, Phys. Rev. C 45, 365 (1992).

[37] S. E. Koonin and J. Randrup, Nucl. Phys. A 474, 173 (1987).

[38] J. E. Finn et al., Phys. Rev. Lett. 49, 1321 (1982).

[39] F. London, Superfluids (John Wiley \& Sons, New York, 1954), Vol. 2.

[40] K. C. Chase and A. Z. Mekjian, Phys. Lett. B 379, 50 (1996). 
[41] D. Stauffer and A. Aharony, Introduction to Percolation Theory, 2nd ed. (Taylor \& Francis, London, 1992).

[42] K. C. Chase and A. Z. Mekjian, Phys. Rev. C 52, R2339 (1995). 


\section{FIGURES}

FIG. 1. Pressure (a) and (in)compressibility (b) of the model at various temperatures.

FIG. 2. Gibbs free energy per particle of the model as a function of pressure at various temperatures.

FIG. 3. Internal energy (a) and specific heat (b) per particle of the model at various densities.

FIG. 4. Entropy per particle of the model at various densities.

FIG. 5. The variance in the nuclear multiplicity of the model as a function of $x^{\prime} / x_{c}^{\prime}$ (a) and $\langle m\rangle / A(\mathrm{~b})$.

FIG. 6. Size of the largest cluster (a) and its variance (b) of the model as a function of $x^{\prime} / x_{c}^{\prime}$. 

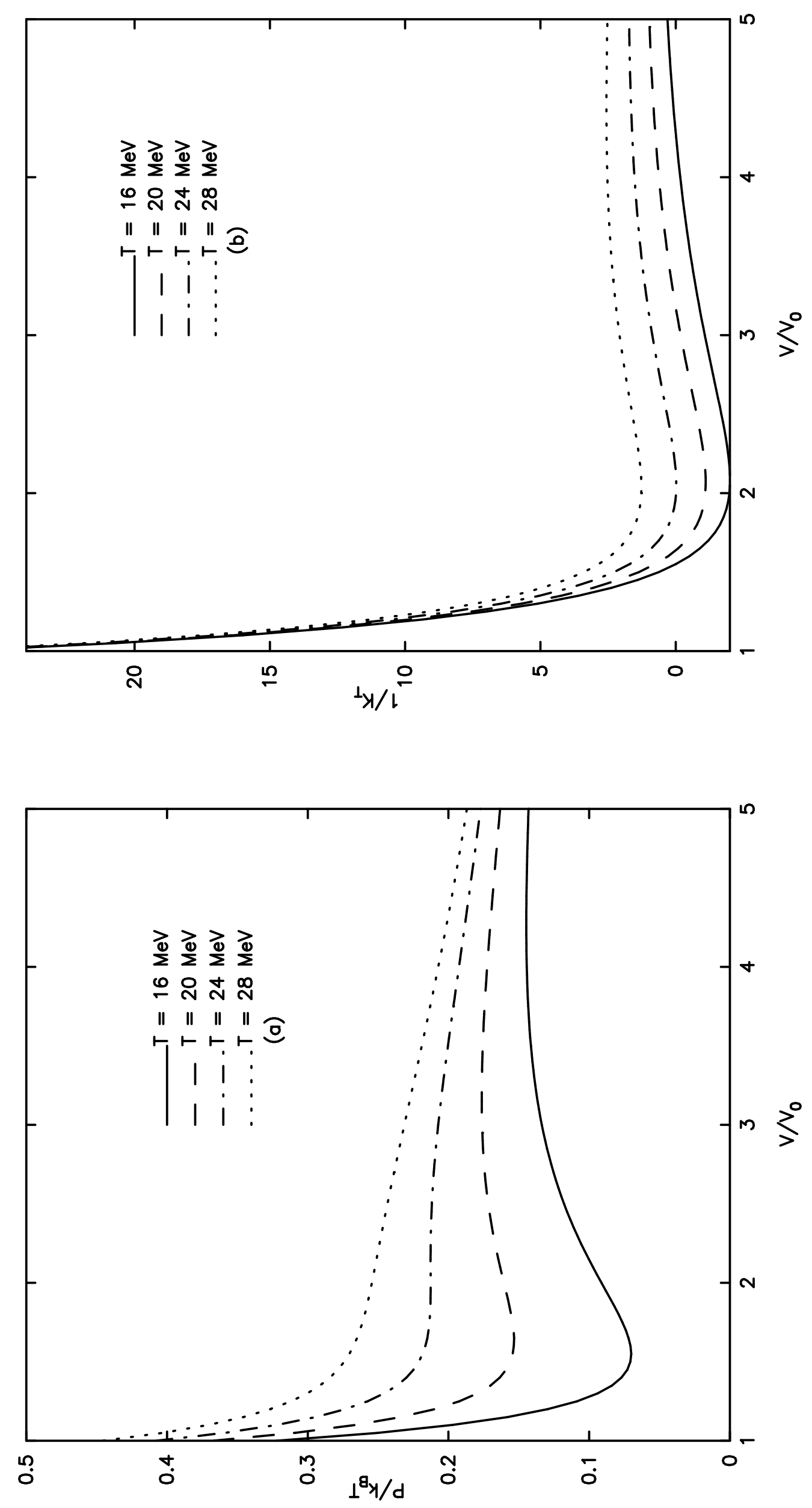


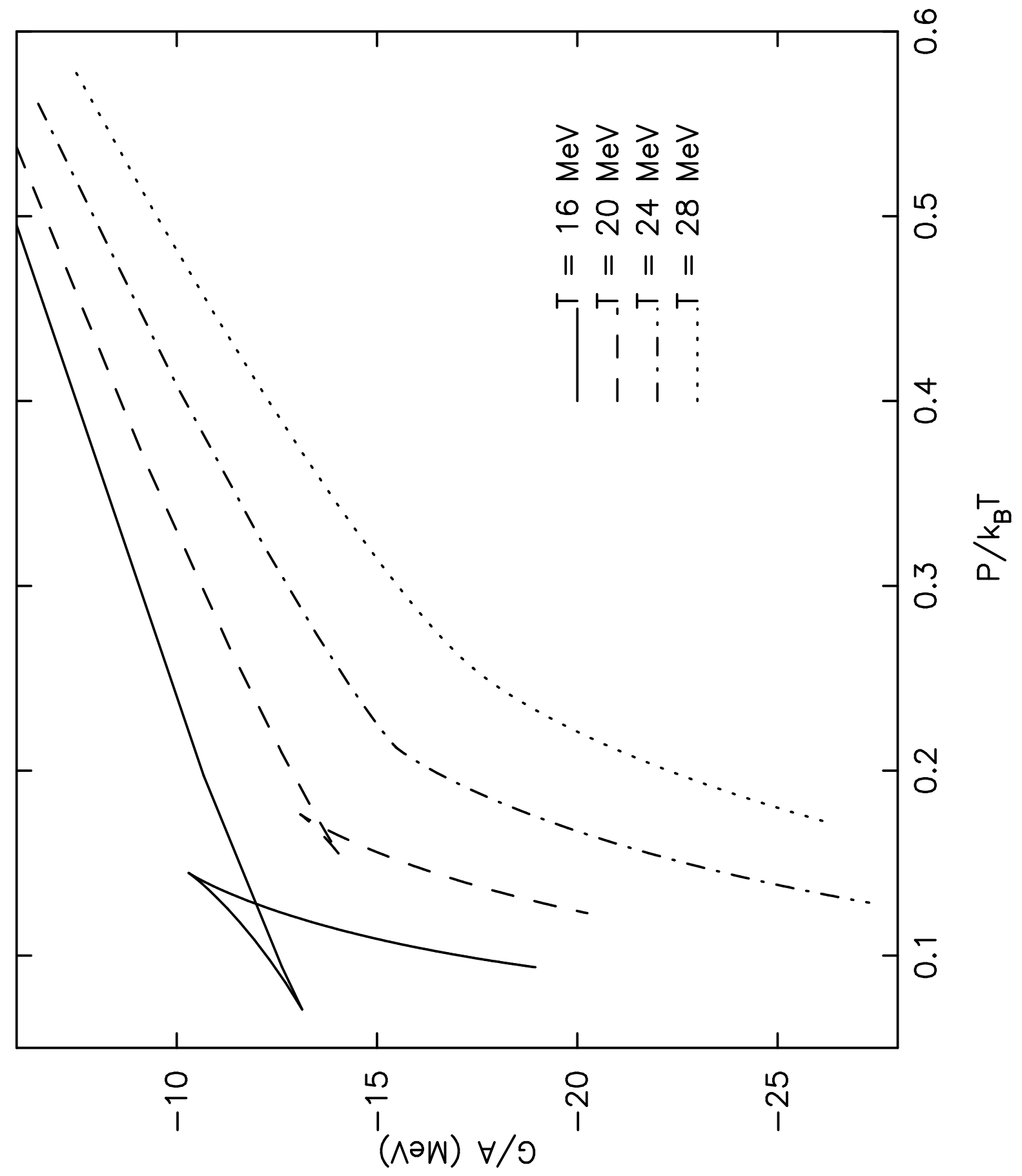



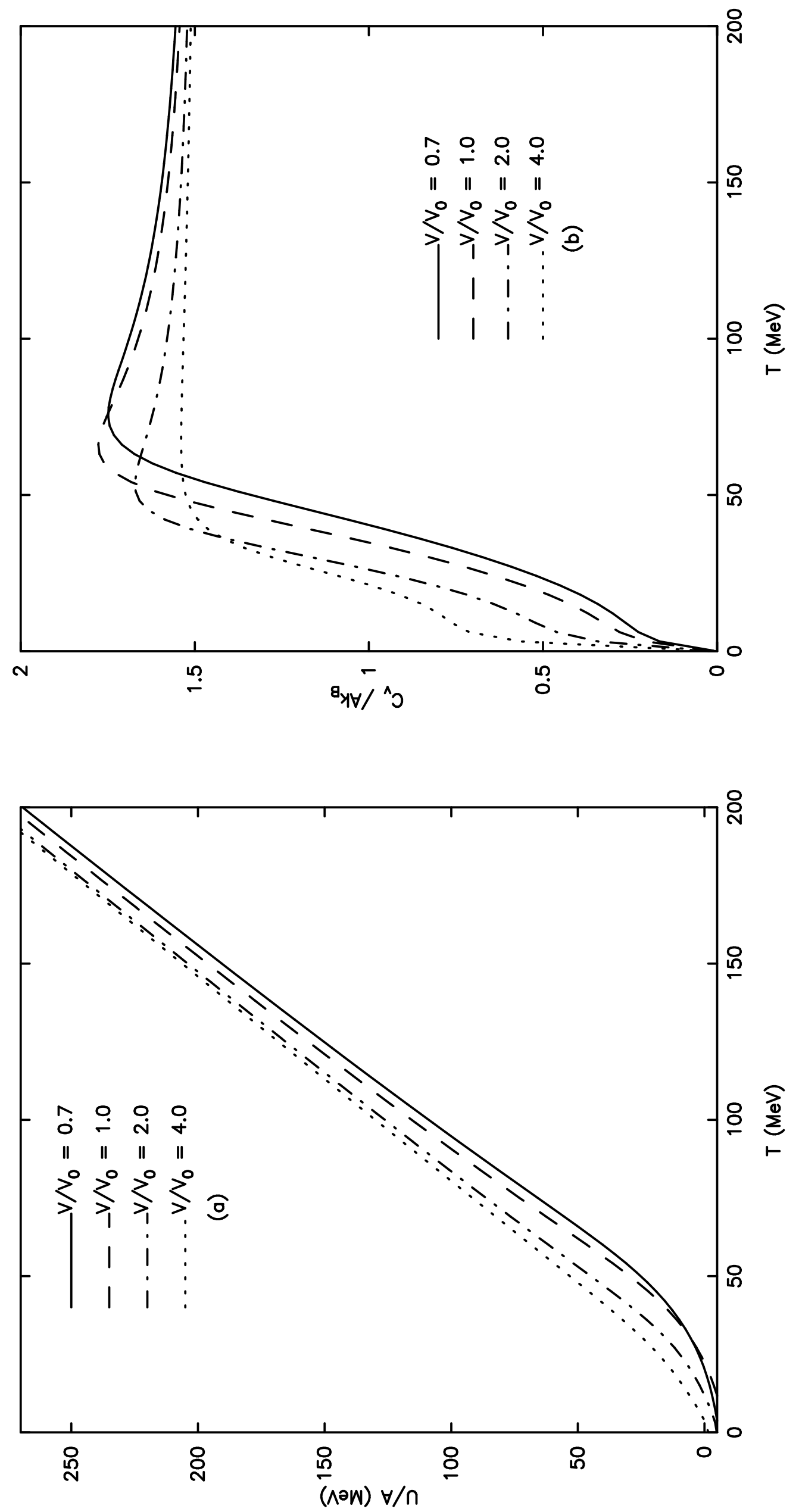


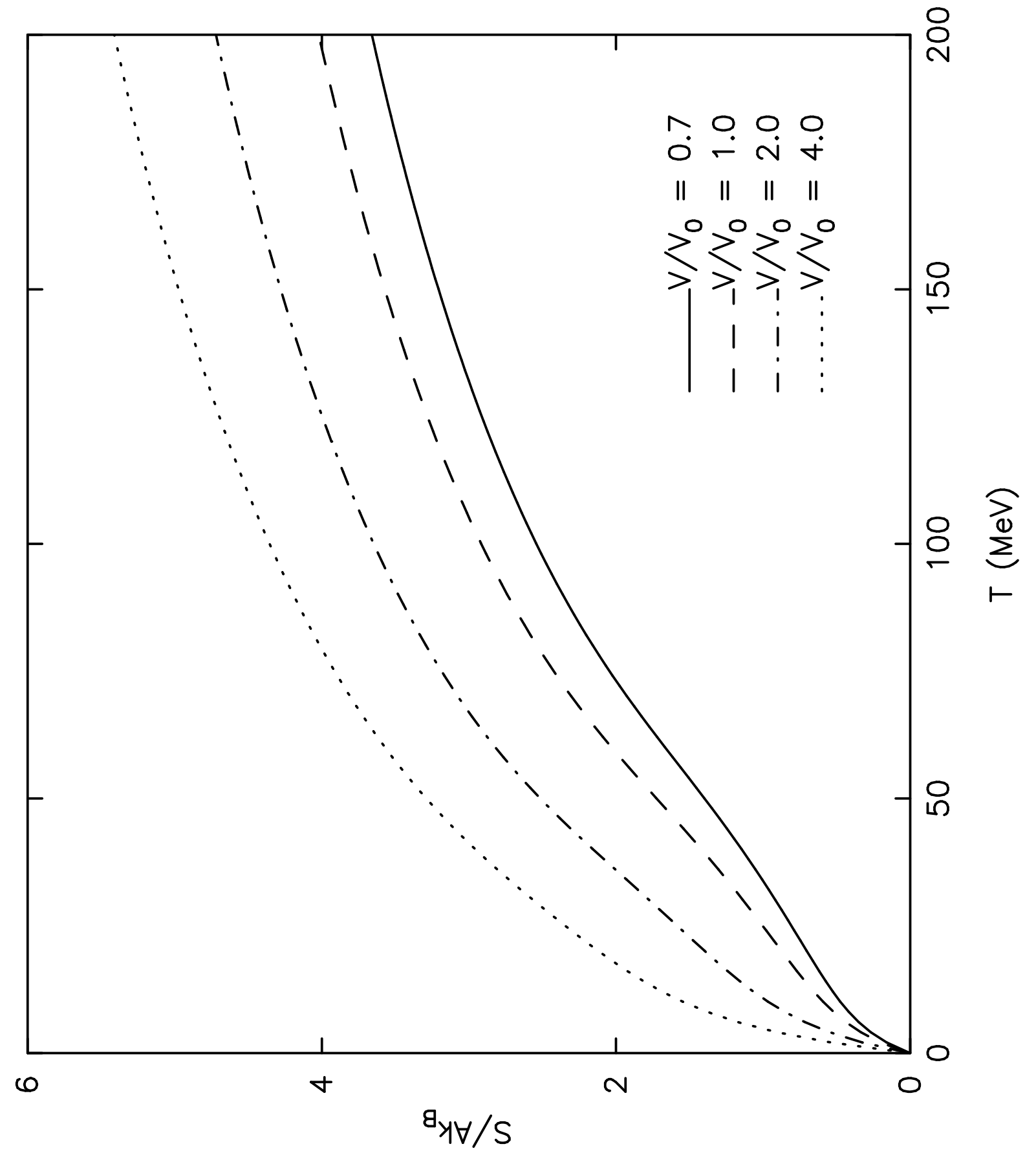



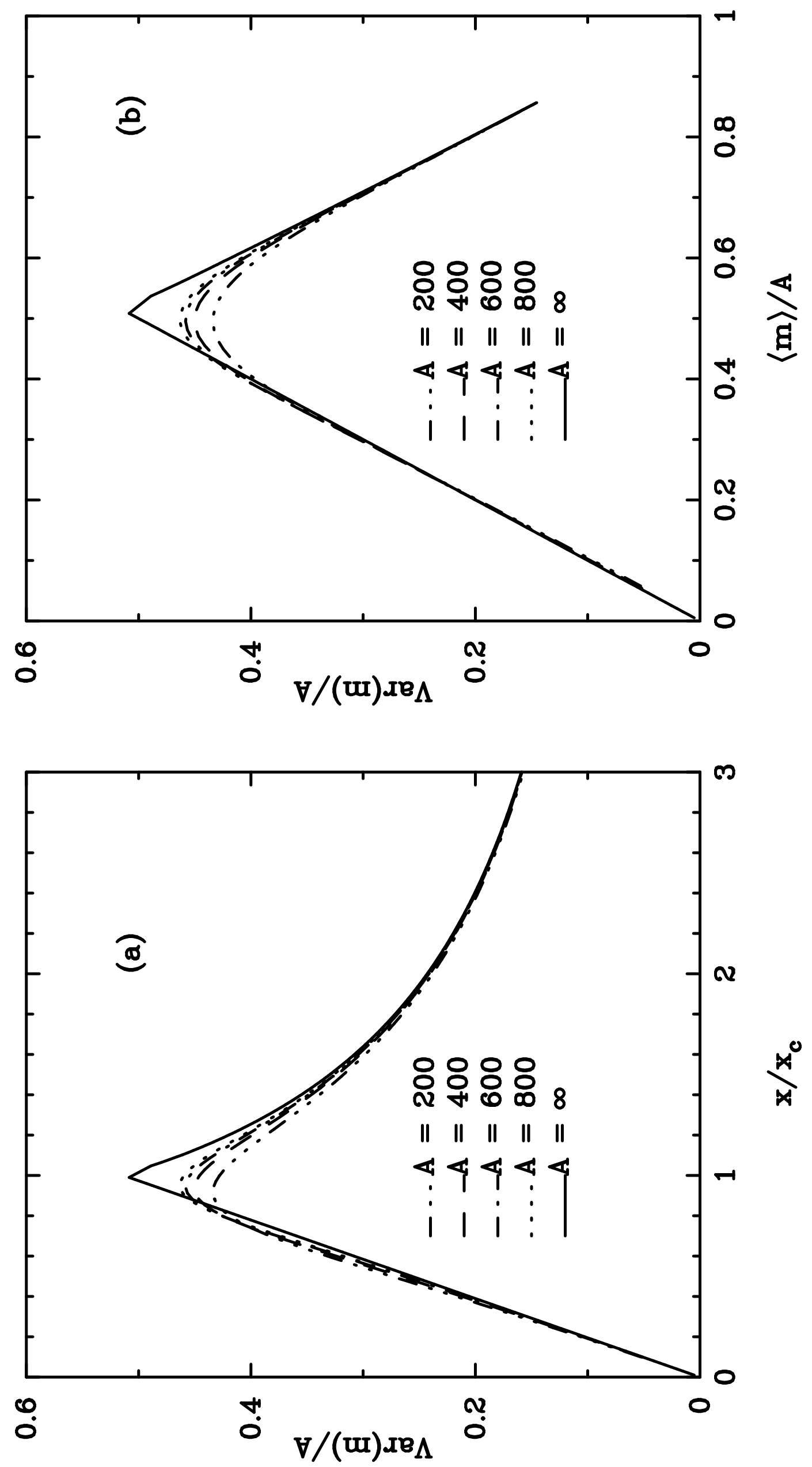

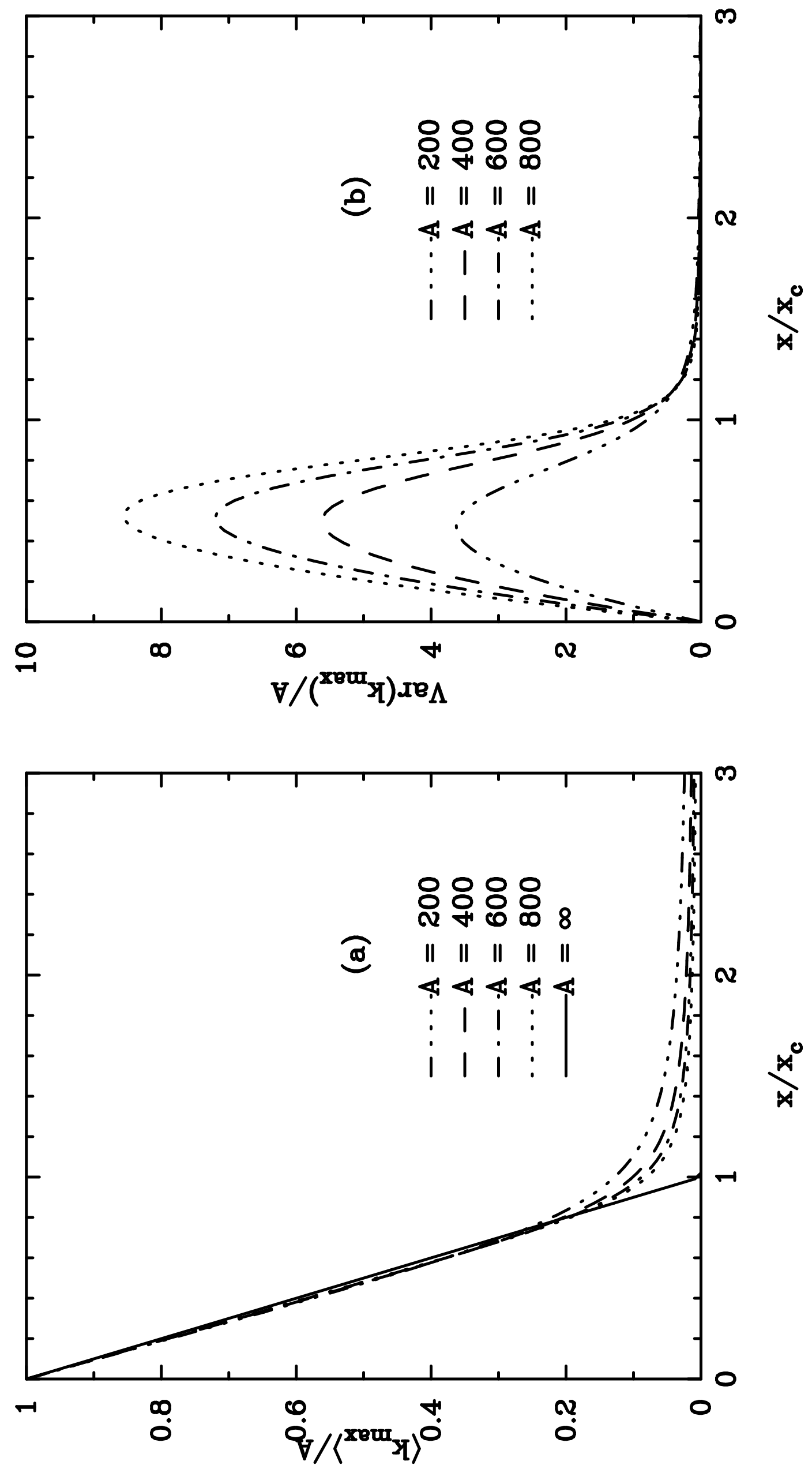